\title{
Chemical Differentiation of Dendrobium officinale and Dendrobium devonianum by Using HPLC Fingerprints, HPLC-ESI-MS, and HPTLC Analyses
}

\author{
Zi Ye, ${ }^{1,2}$ Jia-Rong Dai, ${ }^{3}$ Cheng-Gang Zhang,, ${ }^{1,2}$ Ye Lu, ${ }^{1,2}$ Lei-Lei Wu, \\ Amy G. W. Gong, ${ }^{4}$ Hong Xu, ${ }^{1,2}$ Karl W. K. Tsim, ${ }^{4}$ and Zheng-Tao Wang ${ }^{1,2}$ \\ ${ }^{1}$ The Ministry of Education Key Laboratory for Standardization of Chinese Medicines, The State Administration of \\ Traditional Chinese Medicine Key Laboratory for New Resources and Quality Evaluation of Chinese Medicines, \\ Institute of Chinese Materia Medica, Shanghai University of Traditional Chinese Medicine, Shanghai 201203, China \\ ${ }^{2}$ Shanghai ReD Center for Standardization of Chinese Medicines, Shanghai 201203, China \\ ${ }^{3}$ Longling County Research Institute of Dendrobium, Yunnan 678300, China \\ ${ }^{4}$ Division of Life Science and Center for Chinese Medicine, The Hong Kong University of Science and Technology, \\ Clear Water Bay Road, Kowloon, Hong Kong \\ Correspondence should be addressed to Hong Xu; xuhongtcm@163.com
}

Received 1 March 2017; Revised 9 May 2017; Accepted 18 May 2017; Published 10 July 2017

Academic Editor: Michał Tomczyk

Copyright (C) $2017 \mathrm{Zi}$ Ye et al. This is an open access article distributed under the Creative Commons Attribution License, which permits unrestricted use, distribution, and reproduction in any medium, provided the original work is properly cited.

\begin{abstract}
The stems of Dendrobium officinale Kimura et Migo (Dendrobii Officinalis Caulis) have a high medicinal value as a traditional Chinese medicine (TCM). Because of the limited supply, D. officinale is a high priced TCM, and therefore adulterants are commonly found in the herbal market. The dried stems of a closely related Dendrobium species, Dendrobium devonianum Paxt., are commonly used as the substitute; however, there is no effective method to distinguish the two Dendrobium species. Here, a high performance liquid chromatography (HPLC) method was successfully developed and applied to differentiate D. officinale and D. devonianum by comparing the chromatograms according to the characteristic peaks. A HPLC coupled with electrospray ionization multistage mass spectrometry (HPLC-ESI-MS) method was further applied for structural elucidation of 15 flavonoids, 5 phenolic acids, and 1 lignan in D. officinale. Among these flavonoids, 4 flavonoid C-glycosides were firstly reported in D. officinale, and violanthin and isoviolanthin were identified to be specific for $D$. officinale compared with $D$. devonianum. Then, two representative components were used as chemical markers. A rapid and reliable high performance thin layer chromatography (HPTLC) method was applied in distinguishing $D$. officinale from $D$. devonianum. The results of this work have demonstrated that these developed analytical methods can be used to discriminate $D$. officinale and $D$. devonianum effectively and conveniently.
\end{abstract}

\section{Introduction}

Dendrobium is one of the largest genera among Orchidaceae family plants with more than 1400 species distributed all over the world, and 76 species are found in China [1]. Some Dendrobium species have long been used as precious tonic herbs (Chinese name: $\mathrm{Shi}-\mathrm{Hu}$ ) in traditional Chinese medicine (TCM), which aims to promote the production of body fluids, benefit the stomach, moisten the lungs, and relieve cough [2]. Dendrobium officinale Kimura et Migo (Dendrobii Officinalis Caulis) is one of the most expensive species among medicinal
Dendrobium species. TCM practitioners have widely used D. officinale stems to treat hyperglycemia, hyperlipidemia, chronic gastritis, and dim eyesight and strengthen immunity, as well as an anticancer and antiaging agent, clinically [3]. D. officinale stem is often dried directly or processed into a spiral shape while drying, which is named Tie-Pi-FengDou in Chinese, and is officially recorded in the Chinese Pharmacopoeia [4].

Due to similar morphological characteristics and producing sites, Tie-Pi-Feng-Dou is often confused or mixused with Zi-Pi-Feng-Dou processed by another Dendrobium 
species, D. devonianum, which is mainly produced in Yunnan province. $D$. devonianum stem is recorded officially in local production sites with the same clinical efficiency and usage as Tie-Pi-Feng-Dou [5]. Zi-Pi-Feng-Dou is even purposely named Tie-Pi-Feng-Dou and sold on the market by illegal business behaviors for more profits. This adulteration problem could result in highly variable and inconsistent therapeutic effects in clinical application, which therefore creates a potential health hazard [6]. The stems of D. officinale and D. devonianum have very similar morphological resemblance, and indeed it is difficult to differentiate them just by their appearance. Pharmacological experts and clinical practitioners are often bewildered by these two species when putting their crude drug in use. Unfortunately, there is so far no effective method to identify and distinguish Tie-Pi-Feng-Dou and $\mathrm{Zi}-\mathrm{Pi}-\mathrm{Feng}$-Dou, that is, D. officinale from $D$. devonianum.

Chemical analyses have shown that polysaccharides, phenols, flavonoids, and coumarins are the main compounds isolated from the stems of D. officinale [7] and D. devonianum $[5,8]$. Polysaccharide is the richest ingredient and is considered to be responsible for the pharmacological activities; however, the content of polysaccharide has no obvious difference in D. officinale and D. devonianum stems [9]. Flavonoids are another important compound in D. officinale and $D$. devonianum due to their potential antioxidant, antiinflammatory, and anticancer properties. D. officinale is rich in the flavonoid C-glycoside [7], while D. devonianum is rich in the flavonoid O-glycoside $[10,11]$. HPLC studies focusing on naringenin content [12], fingerprint patterning [13-15], and fragmentation pathways of flavonoids [16] in D. officinale did not provide any distinction between $D$. officinale and $D$. devonianum.

Chromatographic fingerprint comprises one of the important strategies for the quality control of herbal medicines, including identification, and discrimination of some closely related species since they can provide abundant information of complex chemical constituents $[17,18]$. Here, we aimed to develop an effective HPLC method for fingerprinting analysis, which was being applied to distinguish the stems of $D$. officinale and D. devonianum. Specific chemicals, identified by HPLC-ESI-MS ${ }^{n}$, in D. officinale were used as indicative markers for proper identification. In addition, the TLC method of using two flavonoids as chemical markers was developed in differentiating $D$. officinale from $D$. devonianum stems.

\section{Materials and Methods}

2.1. Plant Materials, Chemicals, and Reagents. Twenty batches of stems of $D$. officinale and $D$. devonianum were collected from different parts of China from 2014 to 2016. Five batches of D. officinale (S1-S5) were collected from Mang City (Yunnan province), three batches of D. officinale (S6-S8) were collected from Liancheng County (Fujian province), and two batches of D. officinale (S9-S10) were collected from Yueqing County (Zhejiang province). Ten batches of $D$. devonianum (S11-S20) were collected from Longling County (Yunnan province). All the plant samples were authenticated by one of the authors, Professor Hong Xu. Voucher specimens were deposited at the Institute of Chinese Materia Medica, Shanghai University of TCM, Shanghai, China. Fresh stems were collected and processed into the marketable form, called Feng-Dou. Herbs were ground into powder before the analysis.

Violanthin, isoviolanthin, and vitexin- $2^{\prime \prime}-\mathrm{O}-\beta$-D-glucopyranoside were isolated from the stems of $D$. officinale. Schaftoside and isoschaftoside were purchased from Chengdu Mansite Pharmaceutical Co., Ltd. (Chengdu, China). Rutin and naringenin were obtained from Shanghai $\mathrm{R} \& \mathrm{D}$ Center for Standardization of Chinese Medicine (Shanghai, China). The purities of the abovementioned chemicals were determined to be more than $98 \%$ by normalization of the peak areas detected by HPLC-DAD, as well as by HPLC/MS. HPLC-grade acetonitrile and methanol were purchased from Fisher Chemicals Co. (New Jersey, USA). Analytical-grade ethyl acetate, butanone, and formic acid were purchased from Sinopharm Chemical Reagent Co., Ltd. (Shanghai, China). Deionized water was prepared by a Millipore Milli-Q Plus system (Millipore, Bedford, USA) and used in all experiments.

2.2. Preparation of Standard and Sample Solutions. The references, including violanthin, isoviolanthin, vitexin- $2^{\prime \prime}$ O- $\beta$-D-glucopyranoside, schaftoside, isoschaftoside, rutin, and naringenin, were accurately weighed and dissolved in methanol to obtain a stock standard solution at a concentration of $1 \mathrm{mg} / \mathrm{mL}$, respectively. The solutions were stored at $4^{\circ} \mathrm{C}$ and kept in a dark place. The processed dried stems of $D$. officinale and $D$. devonianum were ground into powder and then passed through a 50-mesh sieve. Powders (2 g) were accurately weighed and immersed in $100 \mathrm{~mL}$ of methanol and extracted in an ultrasonic bath at $25^{\circ} \mathrm{C}$ for $30 \mathrm{~min}$. The supernatants were divided into two equal portions and concentrated, respectively, in vacuo to obtain two brownishblack colored residues. The first residue was redissolved in $1 \mathrm{~mL}$ of methanol and filtered through a $0.45 \mu \mathrm{m}$ membrane as sample solution for HPLC fingerprint and HPLC-ESIMS analysis. The other residue was redissolved in $15 \mathrm{~mL}$ of water, and the solution was extracted by ethyl acetate $(30 \mathrm{~mL})$ three times. The combined water layers were further extracted by butanol $(20 \mathrm{~mL})$ saturated with water three times. The combined butanol layers were concentrated in vacuo, and the residue was redissolved in $1 \mathrm{~mL}$ of methanol and filtered through a $0.45 \mu \mathrm{m}$ membrane as sample solution for HPTLC analysis. In the process of selecting an optimized extraction condition, the extraction method, extraction solvent, extraction time, amount of solvent, and extraction frequency were investigated.

2.3. HPLC Fingerprints Analysis. Samples analysis was carried out on an Agilent 1260 HPLC series liquid chromatography system (Agilent Technologies, Santa Clara, USA), which is equipped with a binary pump, a diode-array detector, an autosampler, and a column temperature controller. Chromatographic separation was performed on a Shiseido Capcell PAK MG-C ${ }_{18}$ column $(250 \times 4.6 \mathrm{~mm} \mathrm{ID,} 5 \mu \mathrm{m}$; Shiseido, Japan) with the column temperature maintained at $30^{\circ} \mathrm{C}$. The mobile phase consisted of acetonitrile (A) and $0.2 \%$ formic 

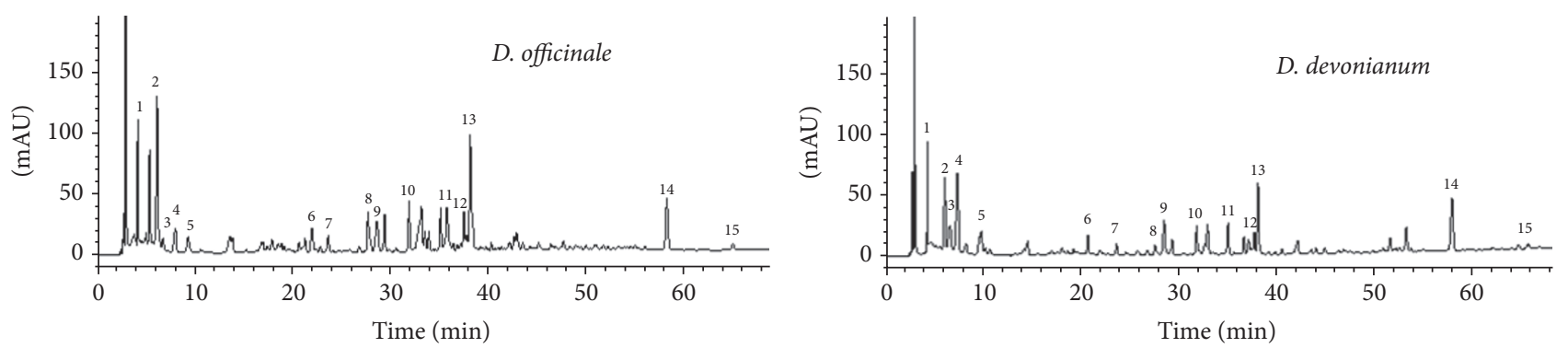

Figure 1: Typical HPLC chromatograms of extracts from dried stems of D. officinale and D. devonianum. Fifteen common peaks were found in D. officinale stems and D. devonianum stems, and obvious differences were shown in $20 \mathrm{~min}$ to $40 \mathrm{~min}$ in their fingerprint, indicating that the HPLC fingerprints could serve as an efficient quality control tool for differentiating D. officinale and D. devonianum.

acid (B) with a linear gradient elution program $(0-5 \mathrm{~min}$, $1 \%-1 \%$ A; 5-15 $\min , 1 \%-10 \%$ A; $15-55$ min, $10 \%-30 \%$ A; $55-65 \mathrm{~min}, 30 \%-35 \% \mathrm{~A}$ ) at a flow rate of $1 \mathrm{~mL} / \mathrm{min}$, and the mobile phase was degassed automatically using an electronic degasser system. The injection volume was $10 \mu \mathrm{L}$. The detector wavelength was set at $270 \mathrm{~nm}$.

2.4. HPLC-ESI-MS Analysis. HPLC-ESI-MS analysis was performed by coupling LC to an electrospray interface and an ion trap analyzer. HPLC separation was carried out by using a Surveyor LC system (Thermo Finnigan, San Jose, CA), with a quaternary pump, continuous vacuum degasser, autosampler, and column compartment, and coupled with a variable wavelength photodiode-array detector. The conditions were the same as those of HPLC fingerprint analysis. The HPLC effluent was introduced into the ESI source in a postcolumn splitting ratio of about $3: 1$. The MS and $\mathrm{MS}^{n}$ analyses were acquired on an LCQ ion trap instrument (Thermo Finnigan, San Jose, CA) equipped with an Xcalibur workstation. The negative ion mode for MS analysis was selected. The operating parameters were optimized as follows: capillary voltage of $5 \mathrm{~V}$, spray voltage of $2.0 \mathrm{kV}$, capillary temperature of $300^{\circ} \mathrm{C}$, sheath gas flow rate at 30 (arbitrary units), and tube lens offset at $20 \mathrm{~V}$. Full-scan mass spectra were recorded in the range $\mathrm{m} / \mathrm{z}$ 150-1000. The isolation width of precursor ions was $1.0 \mathrm{Th}$. The HPLC and LC/MS data were acquired and processed using the Finnigan Xcalibur 1.3 software provided by the manufacturer.

2.5. HPTLC Analysis. $5 \mu \mathrm{L}$ of the extract sample and $1 \mu \mathrm{L}$ of the reference solution were applied on $10 \times 20 \mathrm{~cm}$ HPTLC silica gel $60 \mathrm{~F}_{254}$ plates (Huanghai, China) using a CAMAG (Muttenz, Switzerland) Automatic TLC Sampler (ATS4), which was controlled by Win-CATS software, and were observed by a Reprostar 3 illumination unit. The plate was developed in a twin trough chamber $(10 \times 20 \mathrm{~cm})$ using a solvent system of ethyl acetate/butanone/formic acid/water $(\mathrm{v} / \mathrm{v} / \mathrm{v} / \mathrm{v}, 4: 3: 1: 1)$, sprayed with $1 \%$ aluminium chloride solution after developing, and then dried at $105^{\circ} \mathrm{C}$ for 3 minutes. The plate was examined under ultraviolet light at $366 \mathrm{~nm}$, and the image was taken.

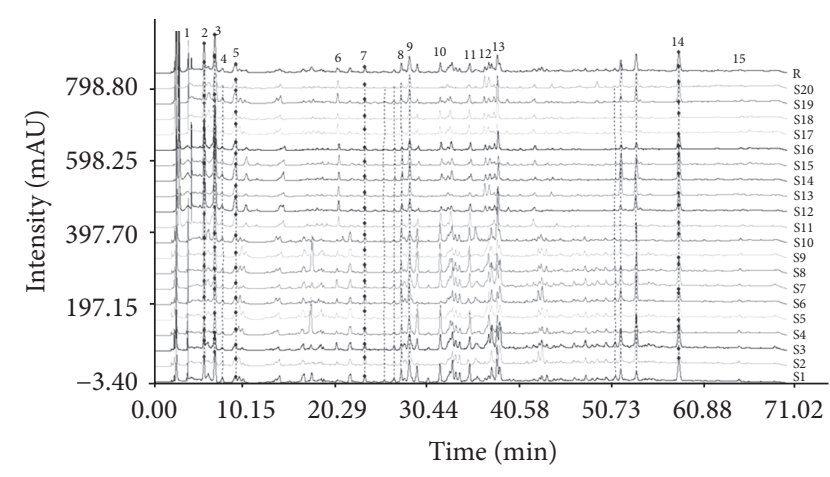

FIGURE 2: The digital standard HPLC fingerprint chromatograms of dried stems of $D$. officinale and $D$. devonianum from different regions of China. Among 15 common peaks (peak 1-peak 15), peak 14 was chosen as the reference peak to calculate RA and RRT of different peaks. D. officinale (S1-S10) and D. devonianum (S11-S20) were marked, and the sources were described in the Materials and Methods.

\section{Results and Discussion}

3.1. HPLC Fingerprint Analysis. The HPLC fingerprint analysis method was validated in terms of specificity, precision, repeatability, and stability according to Chinese Pharmacopoeia [19]. The specificities of 15 common compounds were confirmed by their baseline separation from each other in the chromatograms (Figures 1 and 2). Precision was determined by analyzing the same sample in quintuplicate. The relative standard deviations (RSDs) of relative retention times (RRTs) and relative area (RA) for the 15 common peaks were $0.047-0.608$ and $0.438-3.932 \%$, respectively; their similarities were more than 0.99 . Repeatability tests were performed with 5 independently prepared sample solutions. The RSDs of RRTs and RA of 15 common peaks were 0.027-0.921 and $1.748-3.970 \%$, respectively, and their similarities were also over 0.99 . For the stability test, the same sample solution was analyzed at $0,2,4,8$, and $24 \mathrm{~h}$. The RSDs of RRTs and RA for the 15 common peaks were $0.036-2.123$ and $0.987-3.601 \%$, and their similarities were over 0.99 . These results indicated that the developed method met the technical requirements of fingerprint analysis. The analytical method used in this study 
was reproducible, and the samples were stable during the test period.

Under the established HPLC condition, 10 batches of $D$. officinale stems and 10 batches of $D$. devonianum stems from various sources were analyzed, and the chromatographic fingerprint of each species was obtained, respectively. Figure 1 shows the typical chromatograms of the stem extracts of $D$. officinale and $D$. devonianum. It can be seen that the chromatographic fingerprints of the two species exhibited both similar and different peaks with the 15 common peaks. In D. officinale stem, a complex chromatographic pattern having more characteristic peaks was identified, whereas $D$. devonianum stem showed less characteristic peaks, for example, showing an obvious difference with a high intensity in the $20 \mathrm{~min}$ to $40 \mathrm{~min}$ zone (Figure 1). In order to evaluate the difference and similarity on the stems of $D$. officinale and D. devonianum, 20 chromatograms were grouped together and subjected to Computer Aided Similarity Evaluation System for Chromatographic Fingerprint of TCM (Chinese Pharmacopoeia Commission, 2004 Version), so as to produce a digital standard fingerprint of Dendrobium species (Figure 2). The 15 confirmed common peaks were located in 4 and 70 min running time in all fingerprints of $D$. officinale and $D$. devonianum. Among the 15 common peaks, peak 14 was found to be generally consistent in all 20 chromatograms with reasonable height and resolution. Therefore, this peak (peak 14) was chosen as the reference peak, and RA and RRT of different peaks were calculated. The similarity analysis indicated that the similarity coefficient between $D$. officinale and $D$. devonianum was from 0.343 to 0.903 with an obvious difference, and 10 batches of $D$. officinale had similar HPLC profiles with correlation coefficients in the range of 0.873 to 0.962 , while 10 batches of $D$. devonianum had similar HPLC profiles with correlation coefficients from 0.906 to 0.979 . These data indicated that the chromatograms of $D$. officinale or $D$. devonianum from different sources resembled each other, respectively; however, there were specific differences between $D$. officinale and $D$. devonianum when differentiating between them.

The reliability and accuracy of chromatographic fingerprint combining similarity measure were further addressed by pattern recognition methods, including Principal Component Analysis (PCA) and Partial Least Square Discriminant Analysis (PLS-DA), which are the well-known methods in distinguishing herbal species. They were performed on the 15 common peaks of chromatographic fingerprints with 4 extracted principal components accounting for $83.8 \%$ of the total variance using SIMCA13.0.2 (Umetrics, MKS Instruments Inc., Sweden). The score plot of PCA revealed that the 15 selected common peaks were informative to distinguish chemical differences of $D$. officinale and $D$. devonianum. The 20 samples were densely classified into two major groups, which were clearly differentiated from each other (Figure 3). In the score plot of PLS-DA, the samples were correctly classified into two classes (Figure 4(a)), which was achieved in agreement with the PCA result. Moreover, the variable importance plot (VIP) indicated the variable influence on the classification of samples, showing 8 variable VIP values of peak 7 , peak 4 , peak 9 , peak 12 , peak 15 , peak 2 , peak 3 ,

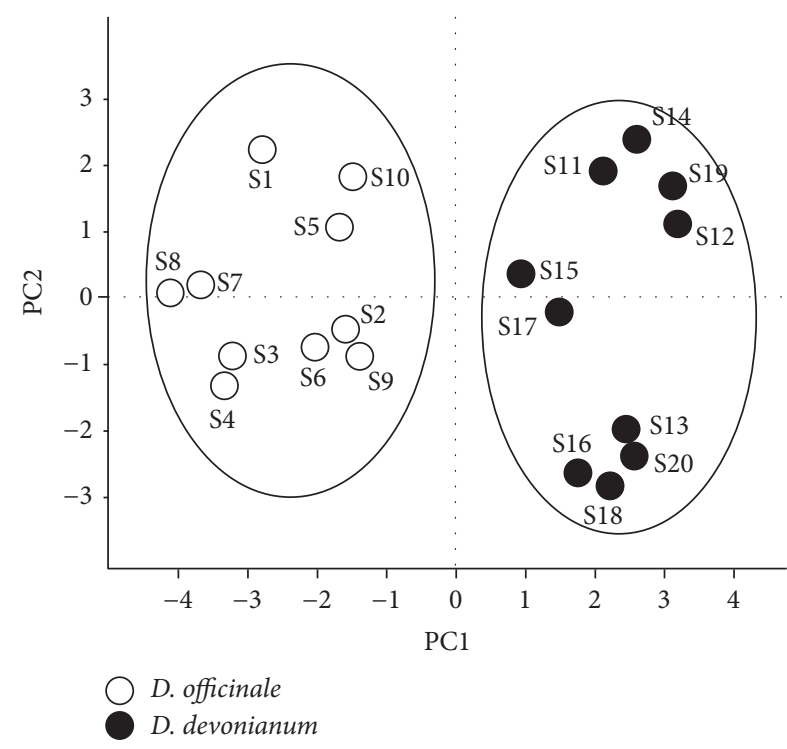

FIGURE 3: The scores plot obtained from PCA analysis of D. officinale and $D$. devonianum. The scores plot of PCA revealed that the 15 selected common peaks were informative to distinguish the chemical difference of $D$. officinale and $D$. devonianum, and the 20 samples were densely classified into two major groups and clearly differentiated from each other.

and peak 8. All VIP values were above 1.0, and all the peaks were significantly different in the two classes $(p<0.05)$ (Figure 4(b)). The results indicated that the 8 aforementioned peaks were the determinants for classification of $D$. officinale and $D$. devonianum stems.

3.2. HPLC-ESI-MS Analysis. In order to identify specific chemicals in $D$. officinale stems, the chemical constituents were characterized from $D$. officinale by HPLC-ESI-MS analysis simultaneously. The structures of compounds (Figure 5) were elucidated according to the molecular weight together with multistage mass fragmentation, retention time, and relative abundance, in comparison with those found in reference compounds and literatures. The chromatogram in negative mode showed better sensitivity than that in positive mode, and a total of 26 compounds were identified, including 14 flavonoids, 5 phenolic acids, 1 lignan, and 6 other compounds (Table 1). Compounds 14, 19, 20, and 22 were reported for the first time in $D$. officinale.

Here, 14 flavonoids were further characterized, 12 of which were identified to be flavonoid glycosides. The MS/MS spectra of these compounds exhibited ions of $m / z[\mathrm{M}-\mathrm{H}-60]^{-}$, $m / z[\mathrm{M}-\mathrm{H}-90]^{-}$, and $m / z[\mathrm{M}-\mathrm{H}-120]^{-}$at different relative abundances, which were demonstrated as characteristic ions of flavonoid C-glycosides [20]. Compound 9 showed the same $[\mathrm{M}-\mathrm{H}]^{-}$ion at $m / z 593$, its MS/MS spectrum produced ions at $m / z 503$ [M-H-90] ${ }^{-}$and $m / z 473$ [M-H-120] $]^{-}$, and it was tentatively identified as 6,8-di-C-glucosyl apigenin according to the literatures $[16,21]$, also named vicenin2. Compounds 10,12, 13, 16, and 24 produced ions at $\mathrm{m} / z$ 563; the fragment ions at $\mathrm{m} / z 503$ [M-H-60] ${ }^{-}, \mathrm{m} / z$ 473 [M-H-90] $^{-}$, and $\mathrm{m} / z 443[\mathrm{M}-\mathrm{H}-120]^{-}$were observed 


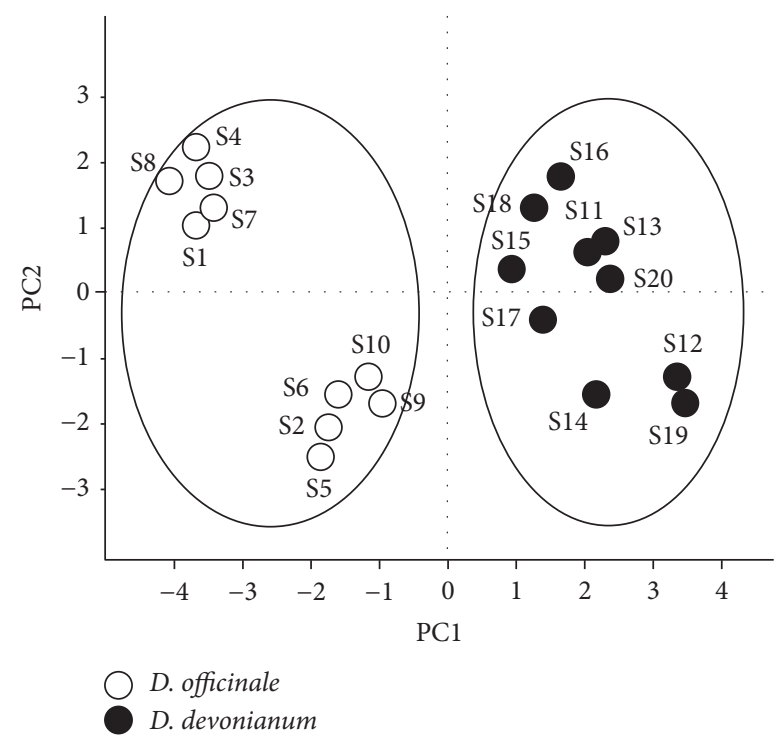

(a)

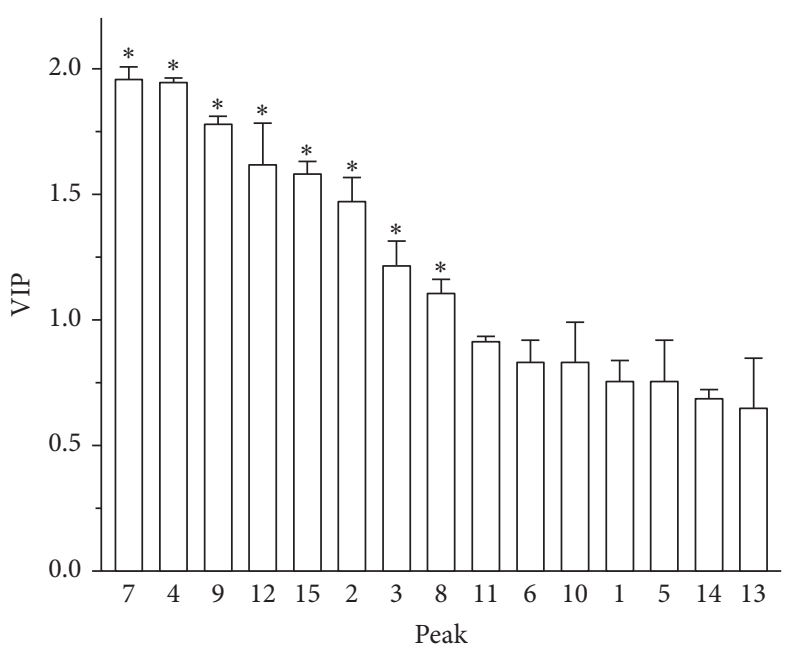

(b)

FIGURE 4: PLS-DA scores plot and VIP (variable importance plot) of D. officinale and D. devonianum. (a) The scores plot of PLS-DA revealed that $D$. officinale and $D$. devonianum could be differentiated according to the 15 selected common peaks. (b) VIP of peak 1 to peak 15 corresponding to the peaks marked in the chromatogram of HPLC fingerprints. Peaks 7, 4, 9, 12, 15, 2, 3, and 8 were all above 1.0; all the peaks were significantly different in the two classes. ${ }^{*} p<0.05$ between $D$. officinale and D. devonianum.

along with the sugar fragment ions (hexose and pentose) in MS/MS. According to the relative abundance of these ions, the position of C-glycosylation was confirmed in C6 or in C-8. They were tentatively assigned as apigenin-6C- $\beta$-D-xyloside- 8 -C- $\beta$-D-glucoside, isoschaftoside, schaftoside, vicenin-3 $[22,23]$, and apigenin-6-C- $\left(2^{\prime \prime}-\mathrm{O}-\beta-\mathrm{D}-\right.$ glucopyranoside)- $\alpha$ - $L$-arabinoside $[24,25]$. Compounds 17 , 18 , and 23 gave the $[\mathrm{M}-\mathrm{H}]^{-}$ions at $m / z 533$, and their $\mathrm{MS}^{2}$ fragment ions showed that their sugars were two pentoses, and thus they were tentatively characterized as apigenin-6C- $\beta$-D-xyloside- 8 -C- $\beta$-D-arabinoside, apigenin-6,8-di-C- $\alpha$ $\mathrm{L}$-arabinoside, and apigenin-6-C- $\alpha$-L-arabinoside- $8-\mathrm{C}-\beta$-Dxyloside [26, 27]. Compounds 14, 19, 20, 22, and 26 were identified as vitexin- $2^{\prime \prime}-\mathrm{O}-\beta$-D-glucopyranoside, violanthin, isoviolanthin, rutin, and naringenin by their retention times and mass spectra with their reference standards, supported by the literatures $[23,27]$.

In the negative mode, the phenolic acids tended to form $[\mathrm{M}-\mathrm{H}]^{-}$ion, followed by the loss $(-44 \mathrm{Da})$ of a carboxylic acid group, and provided an anion of $[\mathrm{M}-\mathrm{H}-\mathrm{COO}]^{-}$. Compound 3 produced the characteristic ion $[\mathrm{M}-\mathrm{H}]^{-}$at $\mathrm{m} / z$ 193.05; the product ions of the $[\mathrm{M}-\mathrm{H}]^{-}$ion gave the fragment ions $\left[\mathrm{M}-\mathrm{H}-\mathrm{CH}_{3}\right]^{-}$at $m / z 175.07$ and $[\mathrm{M}-\mathrm{H}-\mathrm{COO}]^{-}$at $m / z$ 148.95 , and thus compound 3 was assigned as ferulic acid. Compounds 5, 6, 7, and 15 were characterized by the same pattern as dihydroconiferyldihydro- $p$-coumarate, vanillic acid, syringic acid, and $p$-hydroxycinnamic acid [28-34]. The product ions of the $[\mathrm{M}-\mathrm{H}]^{-}$ion (compound 25) at $\mathrm{m} / \mathrm{z}$ 417.02 gave the fragment ions $\left[\mathrm{M}-\mathrm{H}-\mathrm{CH}_{3}\right]^{-}$at $m / z 402.12$, $\left[\mathrm{M}-\mathrm{H}-\mathrm{CH}_{3}-\mathrm{CH}_{3}\right]^{-}$at $m / z$ 387.12, and $\left[\mathrm{M}-\mathrm{H}-\mathrm{C}_{13} \mathrm{H}_{16} \mathrm{O}_{4}\right]^{-}$at $\mathrm{m} / z 181.12$, suggesting the presence of syringaresinol [35]. The corresponding structures of the other 6 fragment ions have not yet been found, and we need to do more research for their identification in the future.

In order to detect the specific compound from $D$. officinale, the same mass spectrometry conditions were applied to analyze the compound difference of $D$. devonianum by selected ion monitoring (SIM) of mass spectrometry scanning mode, in which only a limited mass-to-charge ratio range is detected by the instrument. The results showed that the molecular ion peak of the $[\mathrm{M}-\mathrm{H}]^{-}$ion at $m / z 577.07$ was also found in $D$. devonianum; however, its retention time was $46.16 \mathrm{~min}$ and fragmentation ions in MS/MS were at $\mathrm{m} / \mathrm{z}$ 457.08(4), 413.08(5), 311.08(4), and 269.14(100), which were totally different from those of $[\mathrm{M}-\mathrm{H}]^{-}$ion at $\mathrm{m} / z 577.07$ of violanthin and isoviolanthin in $D$. officinale. Thus, violanthin and isoviolanthin are the specific components of $D$. officinale, which could be used as reference substances to distinguish $D$. officinale from $D$. devonianum. Meanwhile, schaftoside was also identified from the stem of $D$. devonianum, which could be used as a common reference to evaluate $D$. officinale and D. devonianum.

3.3. HPTLC Fingerprints. To investigate the presence and compare the difference of flavonoids in the stems of $D$. officinale and $D$. devonianum by a convenient and effective method, an optimized HPTLC method was further developed and validated with good reproducibility, selectivity, and durability. Using violanthin and schaftoside as reference substances, good separation was attained with the desired $R_{f}$-value range (0.2-0.8) for all bands, 0.26 for schaftoside, and 0.35 for violanthin on a silica gel backed HPTLC plate (silica gel $60 \mathrm{~F}_{254}, 10 \times 20 \mathrm{~cm}$ ) using a mobile phase of ethyl acetate/butanone/formic acid/water (v/v/v/v, $4: 3: 1: 1$ ) 
<smiles>COc1cc(/C=C/C(=O)O)ccc1O</smiles>

3<smiles>COc1cc(CCCOC(=O)CCc2ccc(O)cc2)ccc1O</smiles>

5<smiles>COc1cc(C(=O)O)ccc1O</smiles>

6<smiles>COc1cc(C(=O)O)cc(OC)c1O</smiles>

7<smiles>O=C(O)/C=C/c1ccc(O)cc1</smiles><smiles>[R20]c1c(-c2ccc(O)c(O)c2)oc2cc(O)cc(O)c2c1=O</smiles>

$\mathrm{R}=\mathrm{Rha}-\mathrm{Glc}$

$$
\begin{array}{ll}
9 \mathrm{R}_{1}=\mathrm{Glc} & \mathrm{R}_{2}=\mathrm{Glc} \\
10 \mathrm{R}_{1}=\mathrm{Xyl} & \mathrm{R}_{2}=\mathrm{Glc} \\
12 \mathrm{R}_{1}=\text { Ara } & \mathrm{R}_{2}=\mathrm{Glc} \\
13 \mathrm{R}_{1}=\mathrm{Glc} & \mathrm{R}_{2}=\text { Ara } \\
14 \mathrm{R}_{1}=\mathrm{H} & \mathrm{R}_{2}=\text { Glc-Glc } \\
16 \mathrm{R}_{1}=\mathrm{Glc} & \mathrm{R}_{2}=\mathrm{Xyl}
\end{array}
$$

$$
\begin{array}{ll}
17 \mathrm{R}_{1}=\text { Xyl } & \mathrm{R}_{2}=\text { Ara } \\
18 \mathrm{R}_{1}=\text { Ara } & \mathrm{R}_{2}=\text { Ara } \\
19 \mathrm{R}_{1}=\text { Glc } & \mathrm{R}_{2}=\text { Rha } \\
20 \mathrm{R}_{1}=\text { Rha } & \mathrm{R}_{2}=\text { Glc } \\
23 \mathrm{R}_{1}=\text { Ara } & \mathrm{R}_{2}=\text { Xyl } \\
24 \mathrm{R}_{1}=\text { Ara-Glc } \mathrm{R}_{2}=\mathrm{H}
\end{array}
$$

15<smiles>COc1cc([C@@H]2OC[C@H]3[C@H]2CO[C@H]3c2cc(OC)c(O)c(OC)c2)cc(OC)c1O</smiles>

25 5<smiles>O=C1CC(c2ccc(O)cc2)Oc2cc(O)cc(O)c21</smiles>

22

26

FIgURE 5: Chemical structures of constituents identified in the dried stems of $D$. officinale by HPLC-ESI-MS analysis. (3) Ferulic acid. (5) Dihydroconiferyldihydro- $p$-coumarate. (6) Vanillic acid. (7) Syringic acid. (9) Vicenin-2. (10) Apigenin-6-C- $\beta-D$-xyloside-8-C- $\beta$ D-glucoside. (12) Isoschaftoside. (13) Schaftoside. (14) Vitexin-2" -O- $\beta$-D-glucopyranoside. (15) p-Hydroxycinnamic acid. (16) Vicenin3. (17) Apigenin-6-C- $\beta$-D-xyloside-8-C- $\beta$-D-arabinoside. (18) Apigenin-6,8-di-C- $\alpha$-L-arabinoside. (19) Violanthin. (20) Isoviolanthin. (22) Rutin. (23) Apigenin-6-C- $\alpha$-L-arabinoside-8-C- $\beta$-D-xyloside. (24) Apigenin-6-C-(2" -O- $\beta$-D-glucopyranoside)- $\alpha$ - $L$-arabinoside. (25) Syringaresinol. (26) Naringenin.

[36]. Room temperature and relative humidity at the time of development were $25.0 \pm 2.0^{\circ} \mathrm{C}$ and $45.0 \pm 2.0 \%$, respectively. HPTLC chromatograms of $D$. officinale and $D$. devonianum are shown in Figure 6. The observation at $366 \mathrm{~nm}$ allowed a clear visualization of reference compounds and other bands on the chromatogram, and all bands were well separated, symmetrical, and nontrailed. In 9 batches of $D$. officinale (tracks 1-9), violanthin was easily found with $R_{f}$ value of 0.35 ; however, it was not observed in eight batches of $D$. devonianum (tracks 10-17), while schaftoside $\left(R_{f}=0.26\right)$ was revealed as a common component in both species. The results indicated that violanthin could be a specific compound of $D$. officinale by the developed HPTLC method in differentiating D. officinale and D. devonianum.

\section{Conclusion}

In the present work, an effective HPLC fingerprint analysis method for differentiation and identification of $D$. officinale

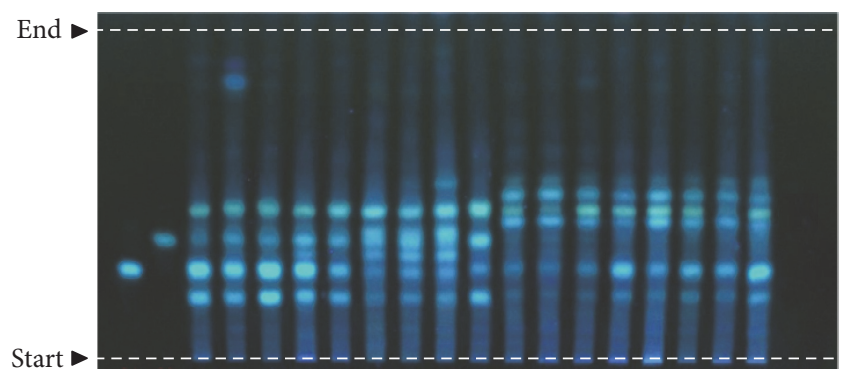

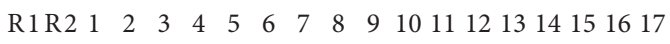

FIGURE 6: HPTLC chromatogram of D. officinale and D. devonianum stems. Schaftoside (R1) and violanthin (R2) were used as markers. Nine batches of $D$. officinale (tracks 1-9) and eight batches of $D$. devonianum (tracks 10-17) were shown.

and $D$. devonianum was developed, with the chromatogram and pattern recognition of HPLC fingerprints: the two species were successfully distinguished from each other. 
TABLE 1: Characterization of compounds from the extract of D. officinale stems by HPLC-ESI-MS (in negative mode). A total of 26 compounds were identified, including 14 flavonoids, 5 phenolic acids, 1 lignan, and 6 other compounds from the stems of $D$. officinale.

\begin{tabular}{|c|c|c|c|c|c|}
\hline Compound & $T_{R} / \min$ & $\begin{array}{l}\mathrm{MS}[\mathrm{M}-\mathrm{H}]^{-} \\
m / z \text { observed }\end{array}$ & $\begin{array}{c}\mathrm{MS}^{2} \\
m / z \text { observed }\end{array}$ & Formula & Identification \\
\hline 1 & 3.20 & 165.07 & $\begin{array}{c}147.02(100) \\
129.00(60), 118.87(27)\end{array}$ & $* *$ & To be identified \\
\hline 2 & 3.25 & 167.04 & $\begin{array}{c}149.08(100) \\
123.29(10), 85.88(13) \\
75.09(80)\end{array}$ & $* *$ & To be identified \\
\hline 3 & 3.67 & 192.96 & 177.09(43), 148.93(51) & $\mathrm{C}_{10} \mathrm{H}_{10} \mathrm{O}_{4}$ & Ferulic acid \\
\hline 4 & 16.84 & 286.91 & $\begin{array}{c}268.91(20) \\
241.00(100), 135.11(10)\end{array}$ & $* *$ & To be identified \\
\hline 5 & 18.86 & 329.01 & $\begin{array}{l}285.16,165.08(100) \\
121\end{array}$ & $\mathrm{C}_{19} \mathrm{H}_{22} \mathrm{O}_{5}$ & Dihydroconiferyldihydro-p-coumarate \\
\hline 6 & 18.74 & 167.05 & $\begin{array}{c}152.03(30) \\
123.07(100), 108.11(5)\end{array}$ & $\mathrm{C}_{8} \mathrm{H}_{8} \mathrm{O}_{4}$ & Vanillic acid \\
\hline 7 & 20.66 & 197.05 & $\begin{array}{c}182.07(100) \\
153.09(50), 137.86\end{array}$ & $\mathrm{C}_{9} \mathrm{H}_{10} \mathrm{O}_{3}$ & Syringic acid \\
\hline 8 & 24.30 & 293.03 & $131.07(100)$ & $* *$ & To be identified \\
\hline 9 & 30.00 & 593.48 & $\begin{array}{c}533.22(8), 503.15(22) \\
473.13(66), 353.17(6)\end{array}$ & $\mathrm{C}_{27} \mathrm{H}_{30} \mathrm{O}_{15}$ & Vicenin-2 \\
\hline 10 & 32.05 & 563.48 & $\begin{array}{c}503.17,473.10(100) \\
443.12(60), 353.14(13)\end{array}$ & $\mathrm{C}_{26} \mathrm{H}_{28} \mathrm{O}_{14}$ & $\begin{array}{l}\text { Apigenin-6-C- } \beta \text { - } D \text {-xyloside- } 8-C-\beta \text {-D- } \\
\text { glucoside }\end{array}$ \\
\hline 11 & 33.68 & 612.84 & $\begin{array}{c}\text { 553(40), 539.16(100), } \\
492.96(40) \\
459.01(30) \\
451.06(50), 293.28(10)\end{array}$ & $* *$ & To be identified \\
\hline 12 & 33.80 & 563.18 & $\begin{array}{c}503.20(60) \\
473.10(100) \\
443.09(64), 353.20(8)\end{array}$ & $\mathrm{C}_{26} \mathrm{H}_{28} \mathrm{O}_{14}$ & Isoschaftoside \\
\hline 13 & 35.93 & 563.58 & $\begin{array}{c}503.20(22) \\
473.17(47), 443.16(41) \\
353.14(7)\end{array}$ & $\mathrm{C}_{26} \mathrm{H}_{28} \mathrm{O}_{14}$ & Schaftoside \\
\hline 14 & 36.57 & 593.67 & $\begin{array}{c}473.10(10) \\
413.12(100), 293.17(6)\end{array}$ & $\mathrm{C}_{27} \mathrm{H}_{30} \mathrm{O}_{15}$ & Vitexin- $2^{\prime \prime}-O-\beta-D$-glucopyranoside \\
\hline 15 & 36.61 & 163.08 & $163.16,119.29$ & $\mathrm{C}_{9} \mathrm{H}_{8} \mathrm{O}_{3}$ & p-Hydroxycinnamic acid \\
\hline 16 & 37.12 & 563.21 & $\begin{array}{c}503(4), 473.17(22) \\
443.13(20), 353.03(6)\end{array}$ & $\mathrm{C}_{26} \mathrm{H}_{28} \mathrm{O}_{14}$ & $\begin{array}{c}\text { Apigenin-6-C- } \beta \text { - } D \text {-glucoside- } 8-C-\beta-D- \\
\text { xyloside }\end{array}$ \\
\hline 17 & 38.04 & 533.58 & $\begin{array}{c}\text { 515.15(30), 473.24(40), } \\
443.15(100) \\
383.08(10)\end{array}$ & $\mathrm{C}_{25} \mathrm{H}_{26} \mathrm{O}_{13}$ & $\begin{array}{l}\text { Apigenin-6-C- } \beta \text { - } D \text {-xyloside- } 8-C-\beta-D- \\
\text { arabinoside }\end{array}$ \\
\hline 18 & 38.38 & 533.17 & $\begin{array}{c}515.18(22), 473.18(40) \\
443.16(80)\end{array}$ & $\mathrm{C}_{25} \mathrm{H}_{26} \mathrm{O}_{13}$ & Apigenin-6,8-di-C- $\alpha$ - $L$-arabinoside \\
\hline 19 & 38.61 & 577.07 & $\begin{array}{c}\text { 559.2(28), 503.02(20), } \\
487.20(50), 473(30) \\
457.15(100) \\
383.20(23)\end{array}$ & $\mathrm{C}_{27} \mathrm{H}_{30} \mathrm{O}_{14}$ & Violanthin \\
\hline 20 & 38.82 & 577.22 & $\begin{array}{c}559.2(42), 503.19(78) \\
473.15(76) \\
457.13(100), 383.15(40)\end{array}$ & $\mathrm{C}_{27} \mathrm{H}_{30} \mathrm{O}_{14}$ & Isoviolanthin \\
\hline 21 & 39.37 & 596.24 & $434.01(100)$ & $* *$ & To be identified \\
\hline 22 & 39.62 & 609.04 & $301.05(100)$ & $\mathrm{C}_{27} \mathrm{H}_{30} \mathrm{O}_{16}$ & Rutin \\
\hline 23 & 41.22 & 533.55 & $\begin{array}{c}515.15(26), 473.16(46) \\
443.15(72)\end{array}$ & $\mathrm{C}_{25} \mathrm{H}_{26} \mathrm{O}_{13}$ & $\begin{array}{c}\text { Apigenin-6-C- } \alpha \text {-L-arabinoside- } 8-C-\beta-D- \\
\text { xyloside }\end{array}$ \\
\hline 24 & 44.03 & 563.10 & 473.13(15), 383.16(100) & $\mathrm{C}_{26} \mathrm{H}_{28} \mathrm{O}_{14}$ & $\begin{array}{l}\text { Apigenin-6-C- }\left(2^{\prime \prime}-\mathrm{O}-\beta-\mathrm{D}-\right. \\
\text { glucopyranoside })-\alpha-L \text {-arabinoside }\end{array}$ \\
\hline 25 & 45.02 & 417.02 & $\begin{array}{l}402.12(40), 387.16(12) \\
181.12(100), 166.09(60)\end{array}$ & $\mathrm{C}_{22} \mathrm{H}_{26} \mathrm{O}_{8}$ & Syringaresinol \\
\hline 26 & 66.21 & 271.23 & 177.15(15), 151.08(80) & $\mathrm{C}_{15} \mathrm{H}_{12} \mathrm{O}_{5}$ & Naringenin \\
\hline
\end{tabular}

$T_{R}$ : retention time. $* *$ : to be identified. 
Using HPLC coupled with ion trap mass spectrometry, a tentative identification of 26 compounds of $D$. officinale could be proposed, including 15 flavonoids, 5 phenolic acids, and 1 lignan. The flavonoids detected were mainly composed of apigenin glycoside derivatives. In addition, violanthin and isoviolanthin were detected in D. officinale whereas they were not detected in D. devonianum. A rapid HPTLC analysis was developed and successfully applied for detecting and identifying the stems of $D$. officinale and $D$. devonianum with their common compound, schaftoside, and specific compounds of violanthin, as in D. officinale. Using violanthin as a specific compound of $D$. officinale, $D$. devonianum could be differentiated by the method of HPTLC. As the results shown here, the developed methods could be used as a combined quality control strategy effectively and efficiently for $D$. officinale and $D$. devonianum.

\section{Conflicts of Interest}

The authors declare that there are no conflicts of interest regarding the publication of this paper.

\section{Acknowledgments}

This research was funded by the Science and Technology Commission of Shanghai Municipality (no. 09405801700; no. 16DZ0500900).

\section{References}

[1] Z. H. Tsi, S. C. Chen, and Y. B. Luo, "Orchidaceae (3)," in Angiospermae, Monocotyledoneae, Flora Reipublicae Popularis Sinica, Z. H. Tsi, Ed., 146, p. 67, Science Press, Beijing, China, 1999.

[2] X. S. Bao, Q. S. Shun, and L. Z. Cheng, The Medicinal plant of Dendrobium (Shi-Hu) in China, Shanghai Medical University Press and Fudan University Press, Shanghai, China, 2001.

[3] L. Chen, P. L. Wei, H. B. Zhou, X. F. Si, C. X. Mu, and M. F. Qiu, "Studies and application of Dendrobium officinale," Asia-Pacific Traditional Medicine, vol. 9, pp. 51-53, 2013.

[4] Chinese Pharmacopoeia Commission, Chinese Pharmacopoeia, vol. 1, Chinese Medical Science and Technology Press, Beijing, China, 2015.

[5] L. L. Cheng, F. J. Yang, H. Y. Wang, W. Li, and M. Li, "Latest research progress on Dendrobium devonianum," Asia-Pacific Traditional Medicine, vol. 11, pp. 31-33, 2015.

[6] J. Xu, Q.-B. Han, S.-L. Li et al., "Chemistry, bioactivity and quality control of Dendrobium, a commonly used tonic herb in traditional Chinese medicine," Phytochemistry Reviews, vol. 12, no. 2, pp. 341-367, 2013.

[7] X. M. Chen, C. L. Wang, J. S. Yang, and S. X. Guo, "Research progress on chemical composition and chemical analysis of Dendrobium officinale," Chinese Pharmaceutical Journal, vol. 48, pp. 1634-1640, 2013.

[8] A. Zhang, "The chemical composition of Dendrobium devoninum Paxt. and its antioxidant activity," China Journal of Chinese Materia Medica, vol. 38, pp. 6-9, 2013.

[9] X.-N. Gan, Y. Xu, H. Xu, J.-B. Liu, L. Yang, and Z.-T. Wang, "Studies on quality evaluation of Dendrobii devoniani," China Journal of Chinese Materia Medica, vol. 38, no. 23, pp. 4113-4118, 2013.
[10] J. Zhou, X. L. Zhou, C. Q. Liang et al., "Chemical constituents of Dendrobium devoniani," Chinese Traditional and Herbal Drugs, vol. 46, pp. 1292-1295, 2015.

[11] Y. Shen, Z. H. Zhou, W. Yang Yao, X. C. Zhao, and A. H. Li, "Chemical constituents from stem of Dendrobium devoniani," Natural Product Research and Development, vol. 24, pp. 339-341, 2012.

[12] G. F. Zhou, S. H. Chen, G. Y. LV, and M. Q. Yan, "Determination of naringenin in Dendrobium officinale by HPLC," China Journal of Chinese Materia Medica, vol. 38, pp. 520-523, 2013.

[13] T. G. He, S. X. Lu, A. Q. Wang, Z. Y. Wei, D. P. Huang, and L. Zhou, "HPLC fingerprint of Dendrobium candidum from Guangxi," Natural Product Research and Development, vol. 24, pp. 353-358, 2012.

[14] J. Cui, L. Yang, S. J. Tan et al., "HPLC fingerprint of Dendrobium officinale," Chinese Pharmaceutical Journal, vol. 48, pp. 16731676, 2013.

[15] M. Q. Yan, S. H. Chen, G. Y. Lv, G. F. Zhou, and X. Liu, "HPLC specific chromatogram of Dendrobium officinale," China Journal of Chinese Materia Medica, vol. 38, pp. 516-519, 2013.

[16] G.-F. Zhou and G.-Y. Lv, "Study on eight flavone C-glycosides in Dendrobium officinale leaves and their fragmentation pattern by HPLC-DAD-ESI-MS ${ }^{n}$," Chinese Pharmaceutical Journal, vol. 47, pp. 13-19, 2012.

[17] P. S. Xie, Y. Z. Yan, B. L. Guo, C. W. K. Lam, S. H. Chui, and Q. X. Yu, "Chemical pattern-aided classification to simplify the intricacy of morphological taxonomy of Epimedium species using chromatographic fingerprinting," Journal of Pharmaceutical and Biomedical Analysis, vol. 52, no. 4, pp. 452-460, 2010.

[18] P. Xia, Z. Bai, T. Liang et al., "High-performance liquid chromatography based chemical fingerprint analysis and chemometric approaches for the identification and distinction of three endangered Panax plants in Southeast Asia," Journal of Separation Science, vol. 39, no. 20, pp. 3880-3888, 2016.

[19] State Food and Drug Administration of China, "Technical requirements for the development of fingerprint of TCM injections," Tech. Rep., SFDA, Beijing, China, 2000.

[20] P. Waridel, J.-L. Wolfender, K. Ndjoko, K. R. Hobby, H. J. Major, and K. Hostettmann, "Evaluation of quadrupole time-of-flight tandem mass spectrometry and ion-trap multiple-stage mass spectrometry for the differentiation of C-glycosidic flavonoid isomers," Journal of Chromatography A, vol. 926, no. 1, pp. 2941, 2001.

[21] Z. Benayad, C. Gómez-Cordovés, and N. E. Es-Safi, "Identification and quantification of flavonoid glycosides from fenugreek (Trigonella foenum-graecum) germinated seeds by LC-DADESI/MS analysis," Journal of Food Composition and Analysis, vol. 35, no. 1, pp. 21-29, 2014.

[22] J. D. Fei, L. Chen, G. Ding et al., "Isolation, identification and quantitative analysis of vitexin $2^{\prime \prime}-O-\beta-D$-glucopyranoside in the whole herb of Flickingeria fimbriata," Chinese Journal of Experimental Traditional Medical Formulae, vol. 19, pp. 4-7, 2013.

[23] F. Ferreres, A. Gil-Izquierdo, P. B. Andrade, P. Valentão, and F. A. Tomás-Barberán, "Characterization of $C$-glycosyl flavones $O$-glycosylated by liquid chromatography-tandem mass spectrometry," Journal of Chromatography A, vol. 1161, no. 1-2, pp. 214-223, 2007.

[24] S. Ding, E. Dudley, S. Plummer, J. Tang, R. P. Newton, and A. G. Brenton, "Fingerprint profile of Ginkgo biloba nutritional supplements by LC/ESI-MS/MS," Phytochemistry, vol. 69, no. 7, pp. 1555-1564, 2008. 
[25] S. Granica and A. K. Kiss, "Secondary metabolites from aerial parts of Circaea lutetiana L," Biochemical Systematics and Ecology, vol. 46, pp. 22-25, 2013.

[26] C. Xie, N. C. Veitch, P. J. Houghton, and M. S. J. Simmonds, "Flavone C-glycosides from Viola yedoensis MAKINO," Chemical and Pharmaceutical Bulletin, vol. 51, no. 10, pp. 1204-1207, 2003.

[27] X. Chen, F. Wang, Y. Wang et al., "Differentiation of the flavonoid C-glycoside isomer in Scuellaria baicalensis Georgi using HPLC-ESI-MS/MS," Chemical Journal of Chinese Universities, vol. 26, pp. 27-30, 2005.

[28] J. Han, M. Ye, X. Qiao, M. Xu, B.-R. Wang, and D.-A. Guo, "Characterization of phenolic compounds in the Chinese herbal drug Artemisia annua by liquid chromatography coupled to electrospray ionization mass spectrometry," Journal of Pharmaceutical and Biomedical Analysis, vol. 47, no. 3, pp. 516-525, 2008.

[29] M.-P. Gonthier, J. L. Donovan, O. Texier, C. Felgines, C. Remesy, and A. Scalbert, "Metabolism of dietary procyanidins in rats," Free Radical Biology and Medicine, vol. 35, no. 8, pp. 837-844, 2003.

[30] Y. Li, C.-L. Wang, F.-F. Wang et al., "Phenolic components and flavanones from Dendrobium candidum," Chinese Pharmaceutical Journal, vol. 45, no. 13, pp. 975-979, 2010.

[31] K.-D. Liu, X. Qiao, Q. Wang, W. Song, D.-A. Guo, and M. Ye, "HPLC-DAD-MSn analysis and HPLC quantitation of chemical constituents in the traditional Chinese medicine formula Yatong-yi-li-wan," Analytical Methods, vol. 5, no. 19, pp. 5241-5247, 2013.

[32] A. R. Rechner, M. A. Smith, G. Kuhnle et al., "Colonic metabolism of dietary polyphenols: influence of structure on microbial fermentation products," Free Radical Biology and Medicine, vol. 36, no. 2, pp. 212-225, 2004.

[33] M. Sanz, B. F. De Simón, E. Cadahía et al., "LC-DAD/ESIMS/MS study of phenolic compounds in ash (Fraxinus excelsior L. and F. americana L.) heartwood. Effect of toasting intensity at cooperage," Journal of Mass Spectrometry, vol. 47, no. 7, pp. 905-918, 2012.

[34] L. M. Bystrom, B. A. Lewis, D. L. Brown, E. Rodriguez, and R. L. Obendorf, "Characterisation of phenolics by LC-UV/Vis, LC-MS/MS and sugars by GC in Melicoccus bijugatus Jacq. 'Montgomery' fruits," Food Chemistry, vol. 111, no. 4, pp. 10171024, 2008.

[35] K. Hanhineva, I. Rogachev, A.-M. Aura, A. Aharoni, K. Poutanen, and H. Mykkänen, "Identification of novel lignans in the whole grain rye bran by non-targeted LC-MS metabolite profiling," Metabolomics, vol. 8, no. 3, pp. 399-409, 2012.

[36] Z. Ye, Y. Lu, Y.-F. Xue, H. Xu, and Z.-T. Wang, "Separation and identification of specific components and quality standard of stem of Dendrobium officinale," China Journal of Chinese Materia Medica, vol. 41, pp. 27-32, 2016. 


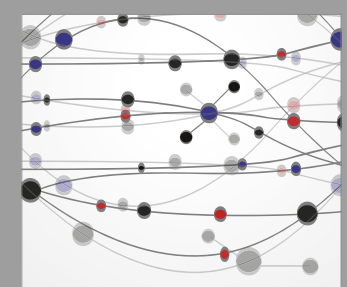

The Scientific World Journal
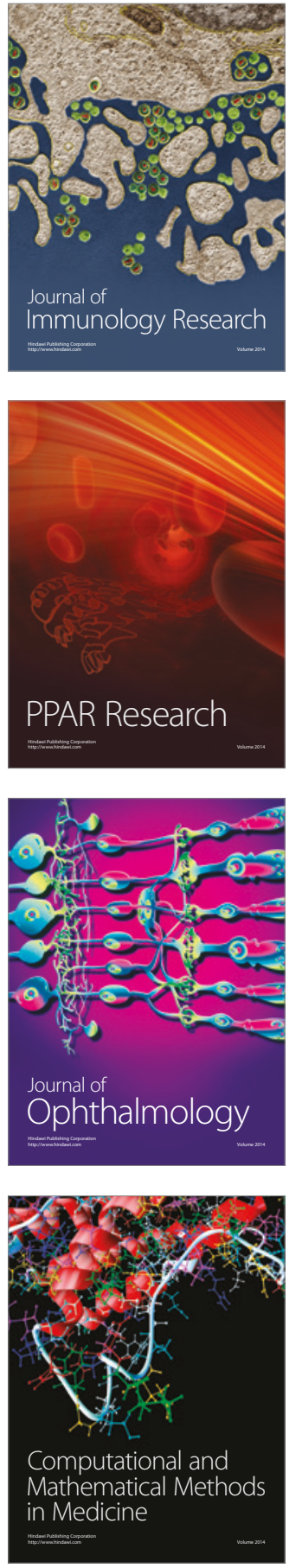

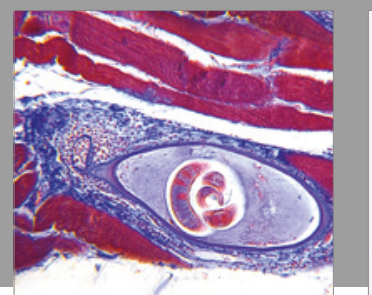

Gastroenterology Research and Practice
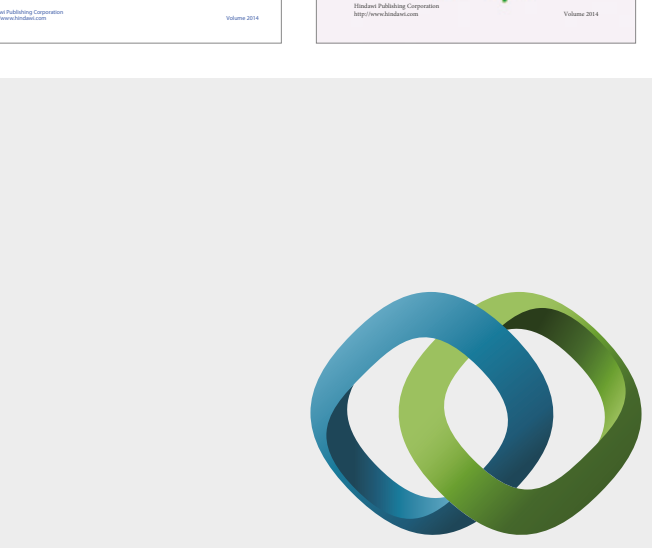

\section{Hindawi}

Submit your manuscripts at

https://www.hindawi.com
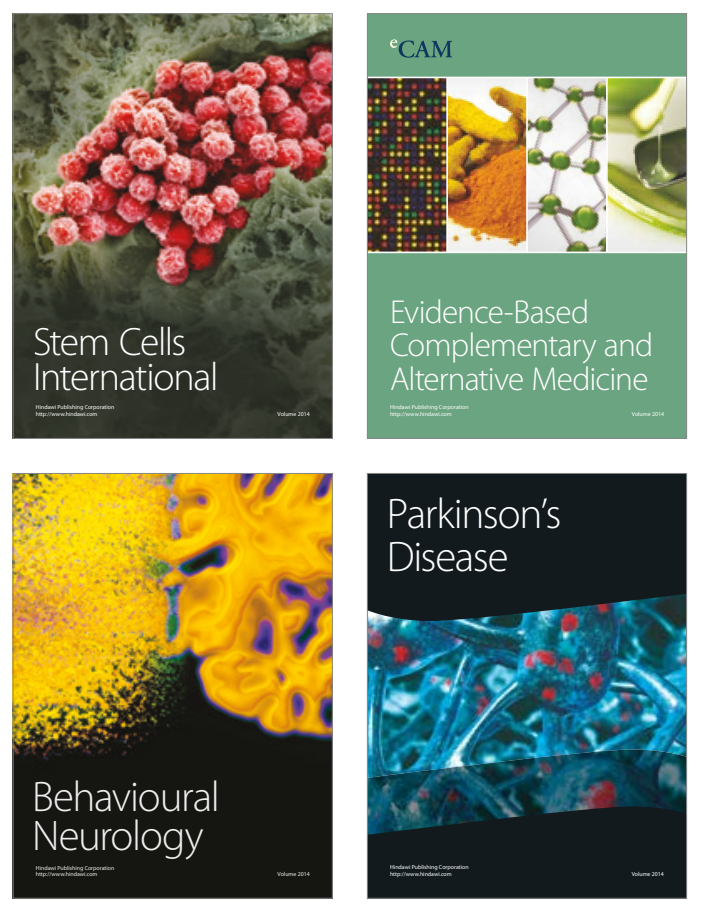
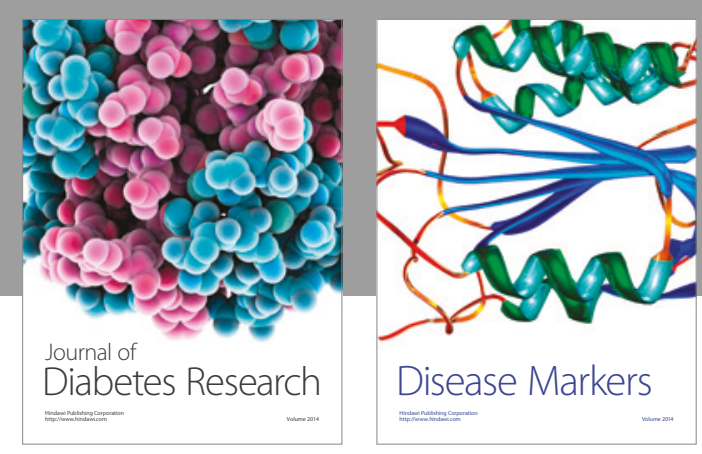

Disease Markers
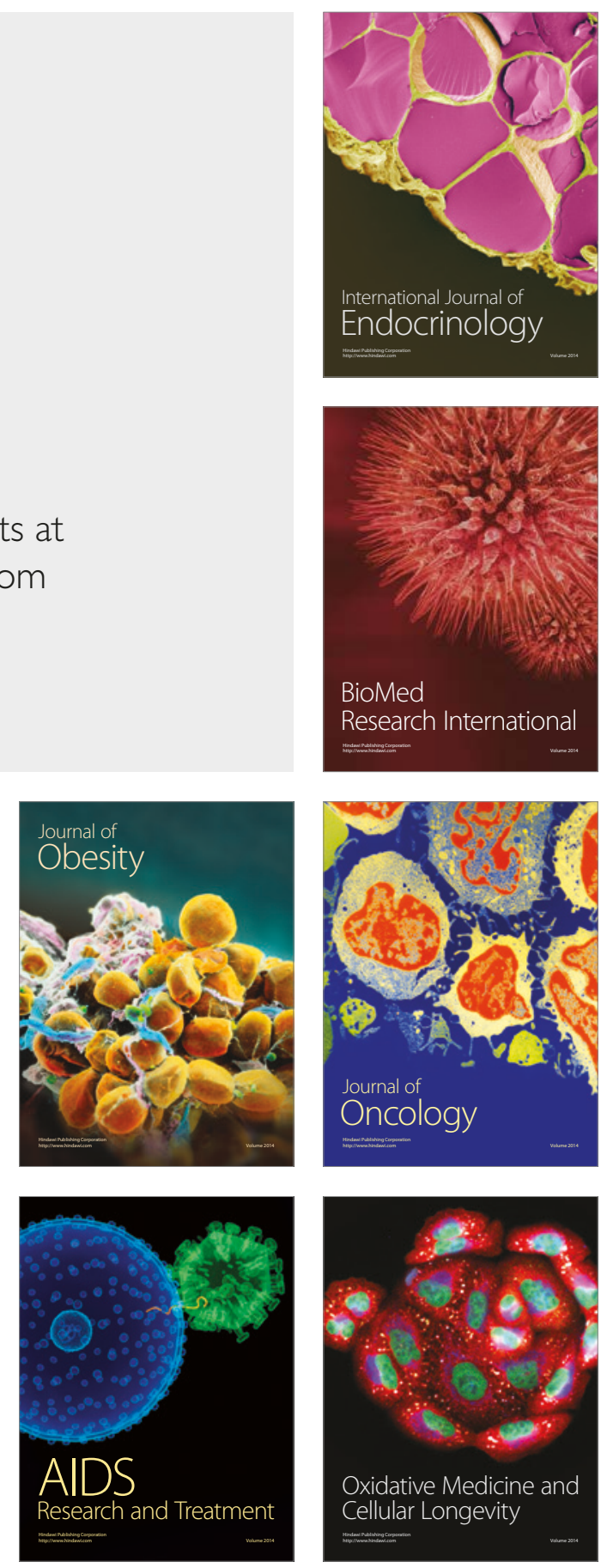